\title{
APLICAÇÃO DA TRANSFORMADA WAVELET DISCRETA NA PREVISÃO DE CARGA A CURTO PRAZO VIA REDES NEURAIS
}

\author{
Agnaldo J. Rocha Reis* \\ Alexandre P. Alves da Silva ${ }^{\dagger}$ \\ * Grupo de Engenharia de Sistemas, Instituto de Eng. Elétrica, Universidade Federal de Itajubá, Av. BPS, 1303 \\ Itajubá, MG, 37500-176, BRASIL \\ ${ }^{\dagger}$ PEE - COPPE - Universidade Federal do Rio de Janeiro, C.P. 68.504 - Rio de Janeiro, RJ, 21945-970, BRASIL
}

\begin{abstract}
The importance of short-term load forecasting has been increasing lately. With deregulation and competition, energy price forecasting has become a big business. Bus-load forecasting is essential to feed analytical methods utilized for determining energy prices. The variability and non-stationarity of loads are becoming worse due to the dynamics of energy prices. Besides, the number of nodal loads to be predicted does not allow frequent interventions from load forecasting experts. More autonomous load predictors are needed in the new competitive scenario. This paper proposes a novel wavelet transform-based technique for short-term load forecasting via neural networks. Its main goal is to develop more robust load forecasters. Two whole years of load data from a North-American electric utility has been used in order to test the proposed methodology.
\end{abstract}

KEYWORDS: Load forecasting, neural networks, power systems, wavelet transform.

\section{RESUMO}

A importância da previsão de carga a curto prazo tem crescido ultimamente. Com a desregulamentação do setor elétrico e a competição advinda desse processo, a previsão do preço de energia se transformou em uma atividade bastante

\footnotetext{
Artigo submetido em 15/12/02

1a. Revisão em 22/04/03

Aceito sob recomendação do Ed. Assoc. Prof. Glauco N. Taranto
}

atraente. A previsão das cargas das diversas barras é essencial para alimentar os métodos analíticos utilizados na determinação dos preços da energia. A variabilidade e a não estacionariedade das cargas estão ficando cada vez mais acentuadas devido à dinâmica das tarifas de energia. Além disso, o número de pontos de carga a serem previstos não permite intervenções freqüentes dos especialistas em previsão. Portanto, previsores de carga mais autônomos são necessários nesse novo cenário competitivo. Este artigo propõe uma nova técnica para a previsão de carga a curto prazo baseada na transformada wavelet discreta. O objetivo principal é desenvolver um previsor neural de carga mais robusto. Dois anos completos de dados de carga de uma concessionária de energia elétrica norte-americana foram utilizados para testar a metodologia proposta.

PALAVRAS-CHAVE: Previsão de carga, redes neurais, sistemas de potência, transformada wavelet.

\section{INTRODUÇÃO}

Redes Neurais Artificiais (RNA) têm sido aplicadas à Previsão de Carga a Curto Prazo (PCCP) com muito êxito (Bakirtzis et al., 1996; Liu et al., 1996; Mohammed et al., 1995; Park et al., 1991). Muitas concessionárias de energia elétrica que anteriormente empregavam ferramentas para a PCCP baseadas em técnicas estatísticas clássicas, estão atualmente utilizando programas para PCCP baseados em RNA (Khotanzad et al., 1998; Mori, 1996). É um daqueles casos raros onde a ciência se transformou em tecnologia após um 
curto período de desenvolvimento. No entanto, essa tecnologia está longe de alcançar o seu limite.

Um importante pré-requisito para uma aplicação bem sucedida das RNA é uma certa regularidade dos dados (Bunn, 2000). Na maioria dos casos, assume-se que uma dada série de carga é estacionária. Para séries de carga elétrica, tal consideração de estacionariedade tem que ser descartada na maioria das vezes. Além disso, deve-se considerar a existência de diferentes tipos de não-estacionariedade.

Para lidar com o problema de não-estacionariedade, wavelets são utilizadas. Isso se deve ao fato das wavelets fornecerem uma boa representação local do sinal tanto no domínio do tempo quanto no domínio da frequiência. Diferentemente das bases de Fourier, as wavelets podem ser mantidas em um intervalo fechado arbitrariamente pequeno (Mallat, 1989). Assim, a transformada wavelet é uma ferramenta muito poderosa para tratar com fenômenos transitórios. Além do mais, a análise de multiresolução via wavelets pode ser utilizada para fornecer uma descrição estatística de cargas elétricas (Misiti et al., 1993).

Neste artigo investiga-se uma estratégia de previsão de carga que explora a propriedade de multiresolução da transformada wavelet. Dois problemas são abordados e tratam da extração de informação relevante de uma curva de carga contaminada com algum tipo de ruído, a saber: 1) Que informação está contida em um sinal, e quais partes dessa informação são úteis para fins de previsão? 2) Há a presença de vários tipos de ruído? É possível identificar cada um deles? Para responder a essas questões, decompõe-se uma série de carga via Transformada Wavelet (TW) em $n+1$ componentes, sendo uma componente de baixa freqüência (ou tendência) mais $n$ componentes de detalhes (onde $n$ é o número de níveis de detalhe). A partir dessa decomposição, duas abordagens são averiguadas. A primeira delas consiste em gerar um modelo de PCCP cujas entradas são compostas por sinais de carga e sinais no domínio de wavelets. A segunda abordagem consiste na modelagem da série de carga no domínio do tempo através da modelagem de cada uma de suas componentes no domínio de wavelets. Para testar a viabilidade das abordagens supraditas, dois anos completos de dados de carga (tomados com base horária) de uma concessionária norte-americana são utilizados ( http://www.ee.washington.edu/class/559/2002spr). As previsões realizadas são do tipo off-line, recursiva e de 1 a 24 passos à frente.

A principal contribuição deste trabalho está relacionada com o aumento da capacidade de generalização de um previsor neural através da extração de características relevantes da série de carga via análise de multiresolução. Uma decomposição em diferentes componentes da série de carga pode ser produzida, o que reduz os esforços de aprendizagem durante o processo de treinamento e validação das RNA (Rocha Reis \& Alves da Silva, 2000).

Este artigo é dividido como se segue. A Seção 2 apresenta a teoria básica sobre a transformada wavelet. Na Seção 3, a análise em multiresolução e o algoritmo piramidal de Mallat são discutidos. A aplicação da Transformada Wavelet Discreta (TWD) na PCCP é assunto da Seção 4. Os modelos de previsão propostos são descritos na Seção 5. Esses modelos são comparados através de simulações na Seção 6. Finalmente, a Seção 7 apresenta as principais conclusões deste artigo e algumas sugestões para trabalhos futuros.

\section{A TRANSFORMADA WAVELET}

A análise via wavelets emprega uma função protótipo chamada wavelet mãe. Essa função tem média zero e decai bruscamente de maneira oscilatória. Os dados são representados via superposição de versões dilatadas e transladadas da wavelet mãe pré-especificada. Matematicamente, a Transformada Wavelet Contínua (TWC) de um dado sinal $x(t)$, com relação a wavelet mãe $g(t)$, é definida como (1), a saber:

$$
T W C(a, b)=\frac{1}{\sqrt{a}} \int_{-\infty}^{+\infty} x(t) g\left(\frac{t-b}{a}\right) d t
$$

em que $a$ é o fator escala e $b$ é o fator de translação.

Um coeficiente TWC(a, b), em uma dada escala e fator de translação, representa o quão bem o sinal original $x(t)$ e a wavelet mãe dilatada/transladada se assemelham. Assim, o conjunto de todos os coeficientes TWC $(\mathrm{a}, \mathrm{b})$, associados com um dado sinal $x(t)$, é a representação wavelet do sinal com respeito a wavelet mãe $g(t)$.

A título de exemplo, considere que o fator de escala de uma wavelet seja igual a $2^{-j}$. À medida que $j$ cresce, o fator de escala decresce, há um "encolhimento" no tempo, as oscilações crescem e a wavelet exibe "alta freqüência". Por outro lado, se $j$ decresce (a escala cresce e há uma expansão no tempo), as oscilações tornam-se mais lentas, i.e., apresentam um comportamento de "baixa freqüência". Esta é uma característica marcante das wavelets - componentes de alta freqüência são analisados em intervalos de tempo curtos enquanto que componentes de baixa freqüência são analisados em intervalos de tempo longos. Os coeficientes de wavelets caracterizam o comportamento local de um sinal. A Fig.1 ilustra algumas famílias de wavelets.

Desde que a TWC é conseguida dilatando-se e transladandose a wavelet mãe continuamente, uma grande quantidade de informação redundante é gerada. Portanto, ao invés de proceder dessa maneira, a wavelet mãe pode ser dilatada e trans- 
(a)

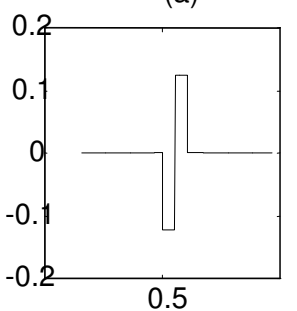

(c)

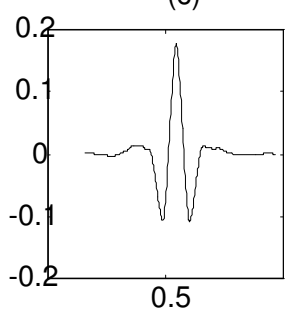

(b)

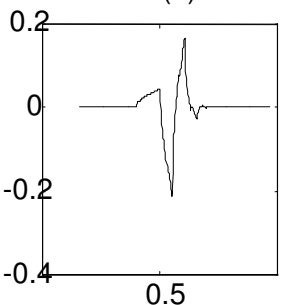

(d)

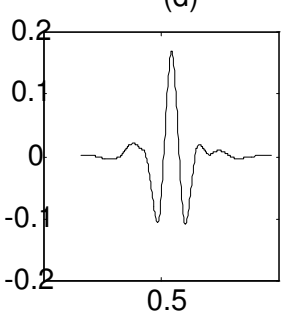

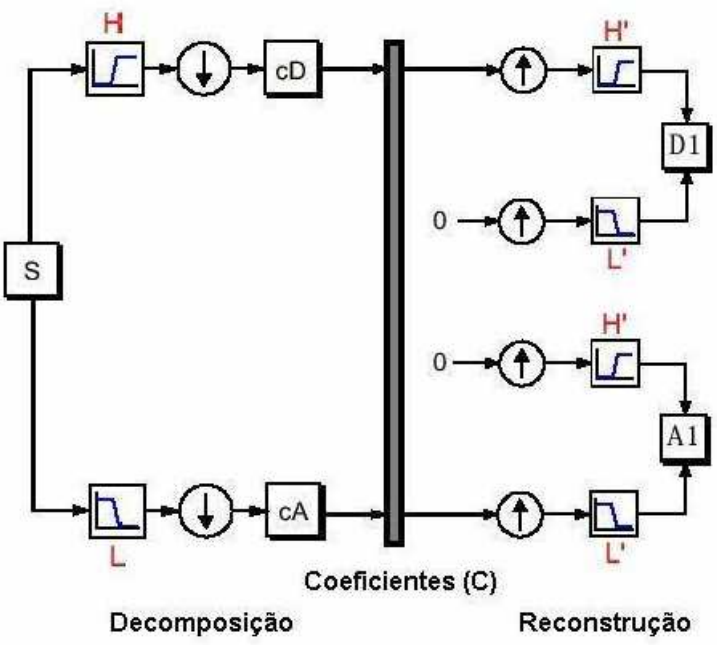

Figura 2: Análise de resolução simples $\left(S=A_{1}+D_{1}\right)$.

Figura 1: Exemplos de wavelet mãe. (a) Haar; (b) Daubechies; (c) Coiflet; (d) Symmlet.

ladada usando escalas e posições especiais baseadas em potência de 2 (i.e, escalas e posições diádicas). Esse esquema é muito mais eficiente e tão eficaz quanto a TWC [Strang \& Nguyen, 1996]. Ele é conhecido como a Transformada Wavelet Discreta (TWD) e definido como (2):

$$
T W D(m, k)=\frac{1}{\sqrt{a_{0}^{m}}} \sum_{n} x(n) g\left(\frac{k-n b_{0} a_{0}^{m}}{a_{0}^{m}}\right)
$$

Os parâmetros $a$ e $b$ (como mostrados em (1)) são funções do parâmetro inteiro $m$, i.e., $a=a_{0}^{m}$ e $b=n b_{0} a_{0}^{m}$. Ainda em (2), $k$ é uma variável inteira que se refere a uma dada amostra no sinal de entrada.

\section{ANÁLISE EM MULTIRESOLUÇÃO E O ALGORITMO PIRAMIDAL DE MALLAT}

Um modo rápido e confiável de se implementar a TWD foi desenvolvido por Mallat em 1989 (Mallat, 1989). A análise de multiresolução via algoritmo piramidal de Mallat referese ao procedimento de se obter "aproximações" e "detalhes" de um dado sinal. Uma aproximação é uma representação de baixa freqüência do sinal original enquanto que um detalhe é a diferença entre duas aproximações sucessivas do sinal original. Uma aproximação mantém a tendência geral do sinal original enquanto que um detalhe mostra os componentes de alta freqüência do mesmo.

O algoritmo piramidal apresenta dois estágios: um para o cálculo da TWD e outro para o cálculo da Transformada Wavelet Inversa (TWI). A multiresolução pode ser obtida

utilizando-se um banco de filtros composto por L, H, L', e H', como mostrado na Fig.2. Os filtros passa-baixa e passaalta de decomposição (L e H), juntamente com os seus filtros de reconstrução associados (L' e H'), formam um sistema chamado Quadrature Mirror Filters (QMF). Uma discussão técnica sobre o projeto e a construção desses filtros pode ser encontrada em (Strang \& Nguyen, 1996).

A partir do sinal $S$ (i.e., $x(n)$ ), dois conjuntos de coeficientes podem ser produzidos a partir da TWD: coeficientes de aproximação $c A l$ e coeficientes de detalhe $c D 1$. Esses conjuntos são obtidos através da convolução de $S$ com o filtro passa-baixa L para aproximação, e com o filtro passa-alta $\mathrm{H}$ para detalhe, seguido por decimação diádica, i.e., a cada duas saídas do filtro, descarta-se uma delas. Esse estágio, conhecido como decomposição (ou análise), determina o conjunto de coeficientes $c A l$ e $c D l$.

Por outro lado, partindo-se de $c A l$ e $c D l$, TWI reconstrói $S$ através da inserção de zeros entre os coeficientes de wavelets, convoluindo-se o sinal resultante com os filtros de reconstrução L' e H'. Esse é o estágio de reconstrução (ou síntese).

Finalmente, um processo de decomposição de múltiplos níveis pode ser conseguido de acordo com a Fig.3. Note que aproximações sucessivas são decompostas de modo que $S$ é dividido em vários componentes de resolução mais baixa.

\section{APLICAÇÃO DA TWD NA PCCP}

Nesta seção encontra-se um exemplo de aplicação da TWD no problema de PCCP. Este exemplo tem como objetivo mostrar a forma típica de saída da decomposição em wavelets de 


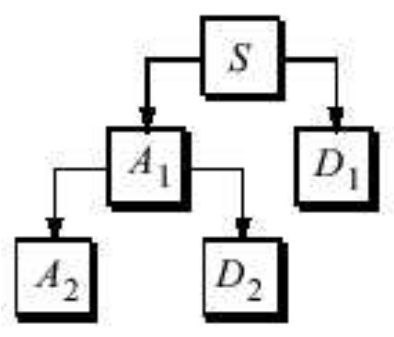

Figura 3: Esquema de decomposição de múltiplos níveis $\left(S=A_{2}+D_{2}+D_{1}\right)$ (Misiti et al., 1996).

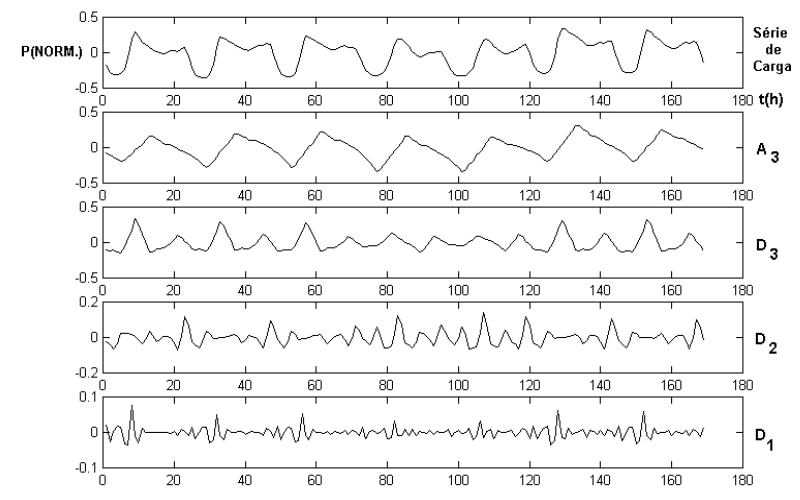

Figura 4: Decomposição em 3 níveis de resolução de uma curva de carga diária.

uma série de carga como uma análise de multiresolução. Para tanto, utilizou-se uma série de carga normalizada entre [-0,5; $0,5]$. Wavelets da família Daubechies foram utilizadas na análise da série. A escolha dessa wavelet-mãe foi baseada em (Misiti et al., 1993). Também, três níveis de resolução foram considerados. Tal nível de detalhamento permite o estudo dos componentes do sinal para os quais o período é inferior a 8h. A Fig.4 ilustra a série de carga considerada, a aproximação $A_{3}$ e os níveis de detalhe $D_{3}, D_{2}$ e $D_{1}$. Com base nessa figura, nota-se que a aproximação $A_{3}$, correspondente a escala de tempo de 8 h, é uma boa aproximação do sinal. Ela representa a sazonalidade diária da carga e é uma versão suavizada da curva de carga. Já os níveis de detalhes $D_{1}$ e $D_{2}$ apresentam valores de baixa magnitude e alguns picos em determinados instantes. Esses sinais exibem um certo tipo de padrão e têm formas e valores médios similares. As irregularidades contidas nesses sinais podem ser causadas por erros de medição e outras fontes de ruído. Finalmente, o nível de detalhe $D_{3}$ apresenta picos que permitem a localização temporal de eventos, tais como, períodos de carga pesada.

\section{MODELOS DE PREVISÃO DE CARGA}

Esta seção descreve os modelos de PCCP considerados neste artigo. Quatro modelos são comparados. O conjunto básico de variáveis de entrada corresponde aos valores em atraso da série de carga horária de $1 \mathrm{~h}, 2 \mathrm{~h}, 24 \mathrm{~h}$ e $168 \mathrm{~h}$, e duas entradas adicionais, $\mathrm{HS}(\mathrm{t})=\operatorname{sen}(2 \pi \mathrm{t} / 24)$ e $\mathrm{HC}(\mathrm{t})=\cos (2 \pi \mathrm{t} / 24)$, codificando a hora do dia $(\mathrm{t})$. A saída corresponde a previsão de carga um passo à frente, que é usada recursivamente para estender o horizonte de previsão até 24 horas.

(Drezga \& Rahman, 1998) mostraram que as melhores variáveis de entrada para previsores de carga lineares não representam, necessariamente, o melhor conjunto de variáveis de entrada para modelos não lineares. Apesar disso, o presente trabalho continua utilizando as variáveis de entrada mais populares para fins de previsão de carga, pois o objetivo principal deste artigo é mostrar que a utilização de wavelets pode influenciar positivamente o desempenho de um previsor neural baseado em Perceptrons de Múltiplas Camadas (PMC).

As arquiteturas selecionadas para cada conjunto de entrada (Subseções de A-D) variam com respeito ao número de neurônios na camada oculta. De um a três neurônios foram utilizados dependendo do modelo em questão (essa escolha foi feita de maneira empírica). Uma única camada oculta foi utilizada. As funções de ativação tangente hiperbólica e linear foram empregadas nas camadas oculta e de saída, respectivamente.

\section{A Modelo 1 (M1)}

O modelo 1 é de certa forma bastante conhecido. Após definir-se a "janela" de dados que será utilizada para fins de treinamento e validação, faz-se a normalização e a padronização da mesma. Esse tipo de pré-processamento faz com que os dados fiquem no intervalo $[-0,5 ; 0,5]$.

A Fig.5 representa esse modelo. Para fins de treinamento, a saída da RNA é a potência ativa no instante de tempo $t$ (i.e., $\mathrm{P}(\mathrm{t})$ ). A arquitetura da rede é composta de 6 entradas, 2 neurônios na camada oculta e 1 neurônio na camada de saída.

\section{B Modelo 2 (M2)}

O modelo 2 é uma combinação de M1 com técnicas de diferenciação. Portanto, duas séries são empregadas: a padronizada e a diferenciada. A diferenciação é aplicada à série padronizada. Para M2, apenas as diferenças de $1^{a}$ ordem (D) são consideradas. As variações de carga foram adicionadas para cada um dos instantes de tempo considerados para a variável carga.

A Fig.6 ilustra o conjunto de entradas de M2. A saída da RNA é a carga medida no instante de tempo $t$ subtraída da 


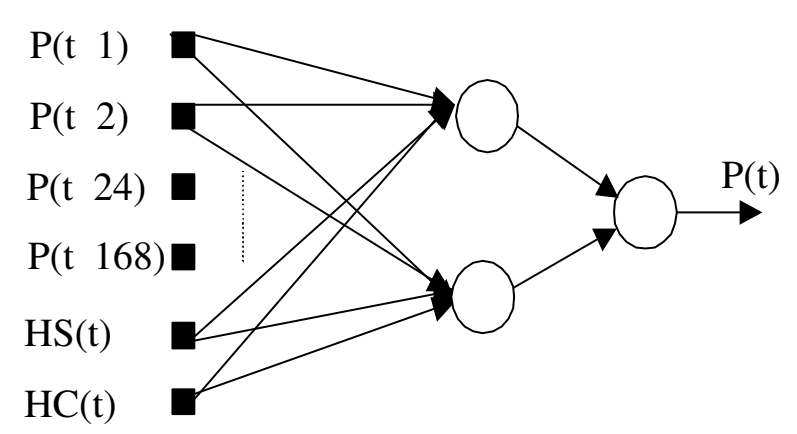

Figura 5: Previsor de carga com entradas básicas.

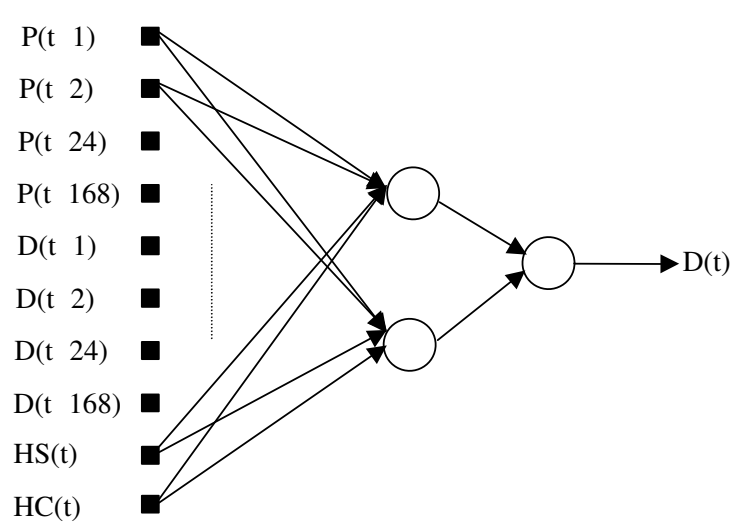

Figura 6: Representação do modelo 2.

carga medida uma hora antes (i.e., $\mathrm{D}(\mathrm{t})=\mathrm{P}(\mathrm{t})-\mathrm{P}(\mathrm{t}-1))$ ). A arquitetura neural consiste de 10 entradas, 2 neurônios na camada oculta e 1 neurônio na camada de saída.

\section{Modelo 3 (M3)}

O modelo 3 (M3) combina normalização, padronização e análise de multiresolução via wavelets. Três níveis de decomposição foram considerados.

Há, basicamente, duas razões principais que justificam a incorporação das wavelets neste modelo. Primeiramente, as características importantes de uma série de carga podem ser enfatizadas. Em segundo lugar, a componente ruidosa da série carga pode ser separada. A Fig. 7 apresenta o esquema de M3.

Como uma tentativa inicial, a aproximação $A_{3}$ e todos os três níveis de detalhes $\left(D_{1}, D_{2}\right.$ e $\left.D_{3}\right)$ foram adicionados ao conjunto de entradas de M2. Com essa configuração, M3 não produziu resultados satisfatórios. Isso já era esperado de alguma forma devido ao fato de que todas as fontes de informação (incluindo informação ruidosa) foram apresentadas a M3.

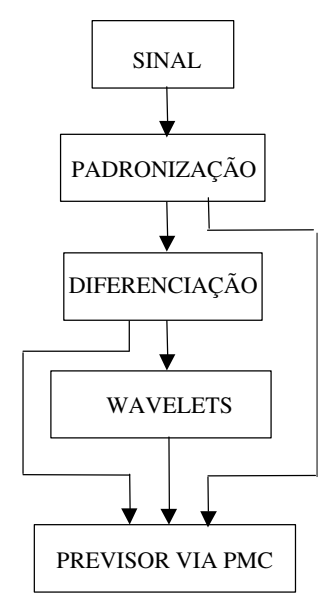

Figura 7: Diagrama esquemático de M3.

Analisando-se os níveis de resolução, constata-se que as subséries mais informativas são $A_{3}$ e $D_{3}$. Já os níveis $D_{1}$ e $D_{2}$ contêm algumas irregularidades que são, via de regra, provenientes de erros de medição e de outras fontes aleatórias de ruído. Essa breve análise sugere que as informações fornecidas pelas subséries $D_{1}$ e $D_{2}$ não devem ser incorporadas a M3.

O conjunto de entradas para M3 pode ser encontrado na Tab.1. A saída da RNA é a carga medida no instante de tempo $t$ subtraída da carga medida uma hora antes (i.e., $\mathrm{CD}(\mathrm{t})=\mathrm{P}(\mathrm{t})$ $\mathrm{P}(\mathrm{t}-1))$. A arquitetura neural consiste de 18 entradas, 3 neurônios na camada oculta e 1 neurônio na camada de saída.

Tabela 1: Variáveis de entrada para M3.

\begin{tabular}{|c|c|c|}
\hline Entrada & Variável & Lags $(\mathrm{h})$ \\
\hline $1-4$ & Carga Diferenciada $(\mathrm{CD})$ & $1,2,24,168$ \\
\hline $5-8$ & Aproximação $\left(\mathrm{A}_{3}\right)$ & $1,2,24,168$ \\
\hline $9-12$ & Carga $(\mathrm{P})$ & $1,2,24,168$ \\
\hline $13-16$ & Detalhe $\left(\mathrm{D}_{3}\right)$ & $1,2,24,168$ \\
\hline 17 & Hora seno & $0^{*}$ \\
\hline 18 & Hora coseno & $0^{*}$ \\
\hline
\end{tabular}

Obs.: O "lag 0" representa o instante alvo.

\section{Modelo 4 (M4)}

O modelo 4 apresenta uma abordagem completamente diferente daquela empregada nos outros modelos. Aqui, a idéia é decompor a série de carga usando-se a análise de multiresolução via wavelets da família Daubechies e modelá-la através do ajuste individual de cada nível de resolução. Isto é, cada componente $\left(A_{3}, D_{3}, D_{2}\right.$ e $\left.D_{1}\right)$ é modelada separadamente e a previsão final é obtida somando-se as quatro previsões realizadas. Note que para M4, a aproximação $A_{3}$ e os três níveis de detalhes devem ser necessariamente levados em conta 


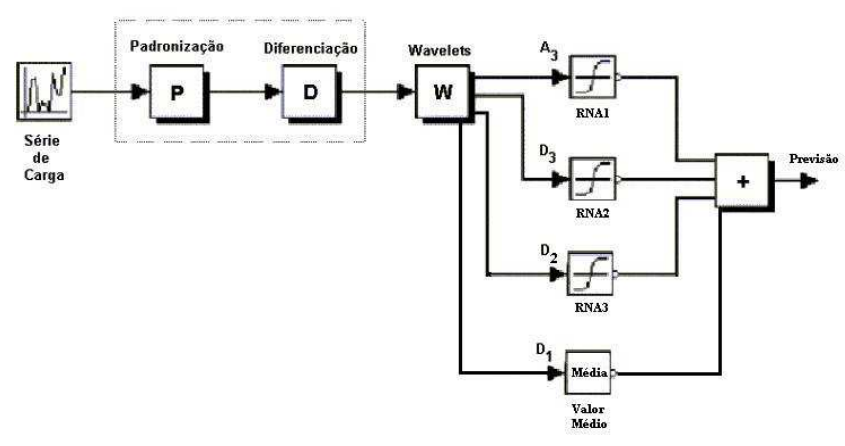

Figura 8: Diagrama esquemático do modelo 4.

para que as previsões possam ser conhecidas. A Fig. 8 mostra o diagrama esquemático desse modelo. Cada um de seus submodelos serão apresentados a seguir.

a) Submodelo para $A_{3}$

Como a aproximação $A_{3}$ é uma versão suavizada da série de carga, as mesmas variáveis de entrada de M1 foram selecionadas (Tab.2). A única diferença é que esse submodelo consiste de 3 neurônios na camada oculta (ao invés de 2) e um neurônio na camada de saída.

b) Submodelos para $D_{3}$ e $D_{2}$

Utilizou-se a função de autocorrelação para selecionar as variáveis de entrada para $D_{3}$ e $D_{2}$. A Tab.2 apresenta o conjunto de entradas e saídas para esses submodelos. Cada uma das redes RNA2 e RNA3 consta de 3 neurônios na camada oculta e 1 neurônio na camada de saída.

c) Submodelo para $D_{1}$

Como o nível de detalhe $D_{1}$ está mais relacionado com a parte ruidosa da série de carga, as previsões para esse nível de resolução são baseadas exclusivamente em valores médios. A média é estimada sobre os dados das janelas de seis semanas para o ano corrente e para os períodos equivalentes - um ano antes e dois anos antes.

\section{TESTES}

Realizou-se uma comparação entre os quatro modelos anteriormente mencionados. Para tanto, utilizou-se um histórico de dados de carga reais observados por uma concessionária de energia elétrica norte-americana no período de janeiro de 1988 a outubro de 1992. Para fins de treinamento e validação dos modelos, janelas de seis semanas do ano corrente, mais janelas de seis semanas do mesmo período dos dois anos anteriores foram utilizadas (e.g., out./nov. 1988, out./nov. 1989 e out./nov. 1990) . O agrupamento de dados foi feito de
Tabela 2: Variáveis de entrada para M4.

\begin{tabular}{|c|c|c|c|c|}
\hline RNA & Entrada & Variável & Lags $(\mathrm{h})$ & Saída \\
\hline \multirow{4}{*}{1} & $1-5$ & Aproximação $\left(\mathrm{A}_{3}\right)$ & $\begin{array}{l}1,2,12, \\
24,168\end{array}$ & \multirow{3}{*}{$\mathrm{A}_{3}(\mathrm{t})$} \\
\hline & $6-10$ & $\mathrm{~A}_{3}$ diferenciada $\left(\mathrm{DA}_{3}\right)$ & $\begin{array}{l}1,2,12 \\
24,168\end{array}$ & \\
\hline & 11 & Hora seno & 0 & \\
\hline & 12 & Hora coseno & 0 & \\
\hline \multirow{5}{*}{2} & $1-5$ & Detalhe $\left(\mathrm{D}_{3}\right)$ & $\begin{array}{l}1,2,12, \\
24,168\end{array}$ & \multirow{5}{*}{$\mathrm{D}_{3}(\mathrm{t})$} \\
\hline & $6-10$ & $\mathrm{D}_{3}$ diferenciada $\left(\mathrm{DD}_{3}\right)$ & $1,2,12$, & \\
\hline & & & 24,168 & \\
\hline & 11 & Hora seno & 0 & \\
\hline & 12 & Hora coseno & 0 & \\
\hline \multirow{5}{*}{3} & $1-5$ & Detalhe $\left(D_{2}\right)$ & $\begin{array}{l}1,2,12, \\
24,168\end{array}$ & \multirow{5}{*}{$\mathrm{D}_{2}(\mathrm{t})$} \\
\hline & $6-10$ & $\mathrm{D}_{2}$ diferenciada $\left(\mathrm{DD}_{2}\right)$ & $1,2,12$, & \\
\hline & & & 24,168 & \\
\hline & 11 & Hora seno & 0 & \\
\hline & 12 & Hora coseno & 0 & \\
\hline
\end{tabular}

acordo com o dia da semana. Para cada dia da semana, um PMC foi treinado com uma variante do algoritmo "backpropagation" com validação cruzada (Masters, 1995). Diferentes partições para os conjuntos de treinamento e validação foram criadas aleatoriamente a cada 150 épocas (90\% dos padrões disponíveis foram utilizados para treinamento). Durante o treinamento, nenhum tratamento especial para feriados foi aplicado.

Depois do treinamento para previsões um passo à frente, previsões recursivas de 1-24 passos à frente são computadas. Os previsores de carga são retreinados no fim do dia. Move-se a janela de treinamento um dia à frente e as previsões para as próximas 24 horas são realizadas (elas se iniciam à meia noite). Esse procedimento de teste é repetido para um período de dois anos completos. Os índices: Erro Percentual Absoluto Médio (MAPE), Erro Quadrático Médio (MSE), Erro Médio (ME) e Erro Máximo (MAX) foram utilizados para avaliar os modelos de previsão de carga. Em linhas gerais, o MAPE permite avaliar, em média, o comportamento de cada um dos modelos. O MSE dá informações a respeito de qual dos modelos apresenta os maiores erros horários. Já o ME informa o quão tendencioso é um dado modelo. Além desses, um índice que quantifica em termos percentuais a melhoria de desempenho de um modelo em relação a outro com respeito ao índice MAPE foi considerado (3).

$$
\text { ganho }(\%)=\left(\frac{M A P E_{\text {anterior }}-M A P E_{\text {atual }}}{M A P E_{\text {anterior }}}\right) \cdot 100 \%
$$

As tabelas que se seguem apresentam os resultados obtidos. A Tab. 3 apresenta uma síntese dos índices de avaliação para os quatro modelos. Já na Tab.4 encontra-se o índice MAPE válido para o horizonte de 1-8 passos à frente. Finalmente, $\mathrm{o}$ MAPE horário da hora $t$ para o conjunto de testes é mostrado 
Tabela 3: Avaliação global dos quatro modelos (1-24 passos à frente).

\begin{tabular}{|c|c|c|c|c|}
\hline Índice & M1 & M2 & M3 & M4 \\
\hline MAPE (\%) & 4,72 & 4,35 & 3,76 & 5,18 \\
\hline MSE $\left(\mathrm{MW}^{2}\right)$ & 23.257 & 19.664 & 15.268 & 32.468 \\
\hline ME (MW) & 5,39 & 16,03 & 5,93 & 27,09 \\
\hline MAX (\%) & 41,88 & 46,76 & 43,10 & 117,12 \\
\hline
\end{tabular}

Tabela 4: MAPE para o horizonte de 1-8 passos à frente.

\begin{tabular}{|c|c|c|c|c|}
\hline Índice & M1 & M2 & M3 & M4 \\
\hline MAPE (\%) & 4,88 & 3,39 & 3,14 & 3,17 \\
\hline
\end{tabular}

na Tab.5. Os testes realizados são conhecidos como "testes cegos", já que os exemplares que formam esse conjunto não participaram das fases de treinamento ou validação dos modelos.

Com base na Tab.3, constata-se que o modelo 3, em média, foi o mais exato. Para múltiplos passos à frente, M3 apresentou erros de previsão significativamente menores que os demais modelos (ver Tab.5). É importante ressaltar que um horizonte de previsão maior não implica necessariamente em um erro de previsão maior. Isso depende da variabilidade dos dados para diferentes períodos do dia e do modelo de previsão adotado. Notar também que o índice MSE, na Tab.3, aponta um número menor de erros elevados para M3 em relação aos demais. M3 também é bastante competitivo para previsões de curtíssimo prazo (vide Tab.4).

Deve-se ressaltar que a inclusão de variáveis climáticas (e.g., temperatura) deve influenciar positivamente o desempenho dos modelos considerados. No entanto, tal informação não foi ainda incorporada nos modelos devido ao fato de que o objetivo do trabalho é verificar o grau de adaptabilidade dos modelos avaliados.

\section{CONCLUSÕES}

Com o crescimento dos sistemas de potência e o aumento na sua complexidade, muitos fatores têm influenciado significativamente a geração e o consumo da energia elétrica. Portanto, o processo de previsão tem se tornado mais complexo, o que requer previsores de carga mais precisos. A previsão de carga a curto prazo é essencial para alimentar os métodos analíticos usados na determinação do preço da energia a curto prazo. A variabilidade e a não estacionariedade das cargas elétricas têm crescido devido à dinâmica das tarifas de energia. Previsores de carga mais autônomos e robustos são necessários no novo ambiente competitivo.

Neste artigo, uma nova abordagem para o problema de PCCP via RNA através da utilização da transformada wavelet dis- creta foi discutida. Quatro modelos de previsão foram apresentados e tiveram seus desempenhos avaliados. As previsões realizadas foram do tipo recursiva, de 1 a 24 passos à frente, em modo off-line, realizadas por perceptrons de múltiplas camadas treinados com uma versão modificada do algoritmo de retropropagação de erros (Masters, 1995). Dois anos completos de dados de carga foram utilizados para testar a metodologia proposta.

A combinação entre padronização, diferenciação de primeira ordem e análise de multiresolução via wavelets (M3), em média, foi a mais eficaz para o horizonte de 1-24 h. Observou-se um ganho médio de $13,49 \%$ em relação ao modelo que combinou somente padronização e diferenciação (M2) e 20,34\% em relação a M1. Já a modelagem da série de carga no domínio do tempo através da modelagem de cada uma de suas componentes no domínio de wavelets (M4), produziu resultados bastante satisfatórios no curtíssimo prazo (1-8h à frente). Esse conjunto de resultados sugere que a análise de multiresolução via wavelets pode contribuir para uma melhoria considerável na exatidão das previsões de carga realizadas por arquiteturas neurais.

Pesquisas futuras enfocarão a incorporação de variáveis de entrada climáticas (e.g., temperatura, umidade, etc.) e outras famílias de wavelets deverão ser testadas. Também, a determinação de intervalos de confiança para as previsões obtidas será considerada (Alves da Silva \& Moulin, 2000).

\section{AGRADECIMENTOS}

Os autores agradecem a agência de fomento CNPq pelo suporte financeiro.

\section{REFERÊNCIAS}

Alves da Silva, A. P. \& Moulin, L. S. (2000). Confidence Intervals for Neural Network Based Short-Term Load Fore-casting. IEEE Trans. on Power Systems, vol. 15, no. 4, Nov. 2000, pp. 1191-1196.

Bakirtzis, A. G., Petridis, V., Klartzis, S. J., Alexiadis, M.C. e Maissis, A.H. (1996). A neural network short-term load forecasting model for the Greek power system. IEEE Trans. Power Systems, vol. 11, no. 2, Maio 1996, pp. 858-863.

Bunn, D. W. (2000). Forecasting loads and prices in competitive power markets. Proceedings of the IEEE, vol. 88, $\mathrm{n}^{o}$. 2, Fev. 2000, pp. 163-169.

Drezga, I. \& Rahman, S. (1998). Input variable selection for ANN-based short-term load forecasting. IEEE Trans. Power Systems, vol. 13, no. 4, Nov. 1998, pp. 12381244. 
Tabela 5: MAPE horário para os quatro modelos.

\begin{tabular}{|c|c|c|c|c|}
\hline Hora (t) & $\begin{array}{l}\text { MAPE } \\
\text { M1 (\%) }\end{array}$ & $\begin{array}{l}\text { MAPE } \\
\text { M2 (\%) }\end{array}$ & $\begin{array}{l}\text { MAPE } \\
\text { M3 (\%) }\end{array}$ & $\begin{array}{l}\text { MAPE } \\
\text { M4 (\%) }\end{array}$ \\
\hline 1 & 1,82 & 1,08 & 0,86 & 1,22 \\
\hline 2 & 3,24 & 1,87 & 1,51 & 2,40 \\
\hline 3 & 4,21 & 2,53 & 2,12 & 2,63 \\
\hline 4 & 4,43 & 3,11 & 2,68 & 2,86 \\
\hline 5 & 4,98 & 3,64 & 3,35 & 3,28 \\
\hline 6 & 6,12 & 4,44 & 4,35 & 3,98 \\
\hline 7 & 7,60 & 5,28 & 5,22 & 4,58 \\
\hline 8 & 6,64 & 5,18 & 5,04 & 4,44 \\
\hline 9 & 5,34 & 4,31 & 4,08 & 3,74 \\
\hline 10 & 4,82 & 3,78 & 3,45 & 3,42 \\
\hline 11 & 4,10 & 3,95 & 3,38 & 3,82 \\
\hline 12 & 3,79 & 4,36 & 3,62 & 4,42 \\
\hline 13 & 3,85 & 4,80 & 3,93 & 4,98 \\
\hline 14 & 4,15 & 5,22 & 4,17 & 5,61 \\
\hline 15 & 4,60 & 5,54 & 4,48 & 6,25 \\
\hline 16 & 5,06 & 5,71 & 4,79 & 6,75 \\
\hline 17 & 5,26 & 5,53 & 4,86 & 6,95 \\
\hline 18 & 5,15 & 5,21 & 4,64 & 7,04 \\
\hline 19 & 4,92 & 4,85 & 4,25 & 6,92 \\
\hline 20 & 4,87 & 4,74 & 4,06 & 7,01 \\
\hline 21 & 4,47 & 4,58 & 3,83 & 7,15 \\
\hline 22 & 4,56 & 4,59 & 3,78 & 7,27 \\
\hline 23 & 4,31 & 4,85 & 3,84 & 8,10 \\
\hline 24 & 4,90 & 5,21 & 4,01 & 9,38 \\
\hline
\end{tabular}

Khotanzad, A., Afkhami-Rohani, R. e Maratukulam, D. (1998). ANNSTLF - artificial neural network shortterm load forecaster - generation three. IEEE Trans. Power Systems, vol. 13, no. 4, Nov. 1998, pp. 14131422.

Liu, K., Subbarayan, S., Shoults, R.R., Manry, M.T., Kwan, C., Lewis, F.L. e Naccarino, J. (1996). Comparison of very short-term load forecasting techniques. IEEE Trans. Power Systems, vol. 11, no. 2, Maio 1996, pp. 877-882.

Mallat, S. (1989). A Theory for Multiresolution Signal Decomposition - The Wavelet Representation. IEEE on Pattern Analysis and Machine Intelligence, vol. 11, $\mathrm{N}^{o} .7$, Jul. 1989, pp.674-693.

Masters, T. (1995). Neural, Novel \& Hybrid Algorithms for Time Series Prediction. New York: Wiley, 1995, p. 514.

Misiti, M., Misiti, Y., Oppenheim, G., Poggi, J.-M. (1993). Ondelettes en Statistique et Traitement du Signal. Revue de Statistique Appliquée, vol. XLI, nº. 4, 1993, pp.33-43.
Misiti, M., Misiti, Y., Oppenheim, G., Poggi, J.-M. (1996). Wavelet Toolbox Manual - User's guide. The Math Works Inc., USA, 1996, p. 626.

Mohammed, O., Park, D., Merchant, R., Dinh, T., Tong, C., Azeem, A., Farah, J. e Drake, C. (1995). Practical experiences with an adaptive neural network short-term load forecasting system. IEEE Trans. Power Systems, vol. 10, no. 1, Fev. 1995, pp. 254-265.

Mori, H. (1996). State-of-the-art overview on artificial neural networks in power systems, In A Tutorial Course on Artificial Neural Networks with Applications to Power Systems. IEEE Catalog no. 96TP112-0, Edited by M.A. El-Sharkawi and D. Niebur, 1996, pp. 51-70.

Park, D.C., El-Sharkawi, M.A., Marks II, R.J., Atlas, L.E. e Damborg, M.J. (1991). Electric load forecasting using an artificial neural network. IEEE Trans. Power Systems, vol. 6, no. 2, Maio 1991, pp. 442-449.

Rioul, O. \& Vetterli, M. (1991). Wavelets and Signal Processing. IEEE Signal Processing Magazine, Out. 1991, pp.14-38.

Rocha Reis, A. J. \& Alves da Silva, A. P. (2000). Préprocessamento de séries temporais para a previsão de carga a curto prazo via redes neurais. Anais do XIII Congresso Brasileiro de Automática, pp. 1138-1143, Florianópolis, SC, Brasil, 2000.

Strang, G. \& Nguyen, T. (1996). Wavelets and Filter Banks. Wellesley-Cambridge Press, Wellesley, 1996, 490 p. 